\title{
Determining quantum Monte Carlo simulability with geometric phases
}

\author{
Itay Hen* \\ Department of Physics and Astronomy and Center for Quantum Information Science \& Technology, \\ University of Southern California, Los Angeles, California 90089, USA \\ and Information Sciences Institute, University of Southern California, Marina del Rey, California 90292, USA
}

(Received 18 December 2020; accepted 12 April 2021; published 29 April 2021)

\begin{abstract}
Although stoquastic Hamiltonians are known to be simulable via sign-problem-free quantum Monte Carlo (QMC) techniques, the nonstoquasticity of a Hamiltonian does not necessarily imply the existence of a QMC sign problem. We give a sufficient and necessary condition for the QMC-simulability of Hamiltonians in a given basis: We prove that a QMC simulation will be sign-problem-free if and only if all the overall total phases along the chordless cycles of the weighted graph whose adjacency matrix is the Hamiltonian are zero (modulo $2 \pi$ ). We use our findings to provide a construction for nonstoquastic, yet sign-problem-free and hence QMC-simulable, quantum many-body models. We also demonstrate why the simulation of truly sign-problematic models using the QMC weights of the stoquasticized Hamiltonian is generally suboptimal. We offer a superior alternative.
\end{abstract}

DOI: 10.1103/PhysRevResearch.3.023080

\section{INTRODUCTION}

The concept of stoquasticity [1] is a key definition in both quantum Monte Carlo (QMC) simulations and computational complexity theory. A Hamiltonian is dubbed stoquastic with respect to a given basis if and only if all its off-diagonal elements in that basis are nonpositive. Otherwise it is referred to as nonstoquastic. In complexity theory, the complexity class StoqMA, associated with the problem of deciding whether the ground-state energy of stoquastic local Hamiltonians is above or below certain values, is expected to be strictly contained in the complexity class QMA (quantum Merlin-Arthur), which deals with general local Hamiltonians [1-3]. StoqMA is also an essential part of the complexity classification of local Hamiltonian problems [4].

In the field of QMC simulations [5,6], the partition function of stoquastic Hamiltonians can always be written as a sum of efficiently computable strictly positive weights [7-9]. As a consequence, such Hamiltonians do not suffer from a sign problem [10,11], i.e., from the existence of negative summands, which greatly impede the convergence of QMC algorithms [10-13].

That stoquasticity leads to sign-problem-free (SPF) representations of quantum many-body physical models has served as the main motivation in numerous recent studies that examine the conditions under which various different classes of nonstoquastic Hamiltonians can be unitarily transformed to

\footnotetext{
*itayhen@isi.edu

Published by the American Physical Society under the terms of the Creative Commons Attribution 4.0 International license. Further distribution of this work must maintain attribution to the author(s) and the published article's title, journal citation, and DOI.
}

equivalent stoquastic [14-17] or minimally nonstoquastic [18] representations.

Although the dichotomy between stoquastic and nonstoquastic Hamiltonians is very often convenient, stoquasticity is not (nor was it intended to be) the property that differentiates between Hamiltonians that are efficiently simulable via QMC, or QMC-simulable for short, and those that are not. Here, QMC simulability is meant in the sense that there exists an SPF representation for the partition function of the Hamiltonian in the basis in which it is represented. Specifically, it is important to note that QMC simulability here does not pertain to and is not meant to imply a polynomial-time convergence, or equilibration, of the algorithm. (In addition, it is worth mentioning that the simulability, or nonsimulability, of a given Hamiltonian in one basis does not imply its simulability or the lack thereof in any other basis.)

Even though the stoquasticity of a Hamiltonian implies an SPF decomposition of the partition function, the converse, namely, that nonstoquasticity leads to a sign-problematic representation, is not necessarily true (see, e.g., Refs. [8,18] for examples). A natural question thus arises: What property of a Hamiltonian makes it QMC simulable? In this paper, we answer this question in terms of the geometric phases associated with the chordless cycles of a weighted graph whose adjacency matrix is the Hamiltonian in question. Our result also has practical significance. First and foremost, it clearly illustrates that curing the sign problem of a model, i.e., finding a unitary transformation that produces an SPF representation for it, is markedly different from curing nonstoquasticity (finding unitary transformations that make the Hamiltonian stoquastic). In fact, our result shows that the latter approach, which is the current standard practice [14-18], should not be used toward rendering a Hamiltonian simulable. In addition, our result demonstrates that stoquastization of sign-problematic Hamiltonians, the method normally used for assigning positive weights to QMC configurations is, in 
general, a suboptimal choice; a superior alternative can be given in terms of the geometric phases of the Hamiltonian.

We further demonstrate the above by introducing a class of quantum many-body models that, while being nonstoquastic, are SPF and therefore perfectly simulable by QMC techniques. We proceed to show that all SPF Hamiltonians can be represented by stoquastic Hamiltonians rotated by some diagonal unitary.

The paper is organized as follows. In Sec. II, we present a generic partition function decomposition focusing on the signs of its summands and their origins, which we trace back to geometric phases associated with the graph structure of the Hamiltonian. Based on the observations made in the preceding section, we provide in Sec. III a construction for nonstoquastic SPF Hamiltonians. In Sec. IV, we discuss QMC simulations of sign-problematic Hamiltonians. Conclusions are given in Sec. V.

\section{EMERGENCE OF THE SIGN PROBLEM AS A NONTRIVIAL GEOMETRIC PHASE}

We now examine in detail the origins of the sign problem in QMC simulations. By doing so, we are able to differentiate QMC-simulable Hamiltonians (in a given basis) from nonsimulable ones.

\section{A. Permutation matrix representation of Hamiltonians}

We start our derivation by considering a Hamiltonian $H$ given in a basis $\mathcal{B}=\{|z\rangle\}$, which we refer to as the computational basis, and write the Hamiltonian in permutation matrix representation (PMR) $[9,19,20]$, i.e., as a sum

$$
H=\sum_{j=0}^{M} \tilde{P}_{j}=\sum_{j=0}^{M} D_{j} P_{j}
$$

where $\left\{\tilde{P}_{j}\right\}$ is a set of $M+1$ distinct generalized permutation matrices [21], i.e., matrices with precisely one nonzero element in each row and each column (this condition can be relaxed to allow for zero rows and columns). Each operator $\tilde{P}_{j}$ can be written, without loss of generality, as $\tilde{P}_{j}=D_{j} P_{j}$ where $D_{j}$ is a diagonal matrix and $P_{j}$ is a permutation matrix with no fixed points (equivalently, no nonzero diagonal elements) except for the identity matrix $P_{0}=1$. We will call the diagonal matrix $D_{0}$ the classical Hamiltonian. Each term $D_{j} P_{j}$ obeys $D_{j} P_{j}|z\rangle=d_{z^{\prime}}^{j}\left|z^{\prime}\right\rangle$, where $d_{z^{\prime}}^{j}$ is a possibly complex-valued coefficient and $\left|z^{\prime}\right\rangle \neq|z\rangle$ is a basis state. We note that casting physical (e.g., $k$-local) Hamiltonians in PMR form can always be done efficiently [9].

\section{B. Partition function decomposition}

Having cast $H$ in PMR form, we next derive an expression for the partition function $Z=\operatorname{Tr}\left[e^{-\beta H}\right]$. Expanding the exponential in a Taylor series in the inverse temperature $\beta, Z$ can be written as a triple sum over all basis states $|z\rangle$, the expansion order $q$ which ranges from 0 to infinity, and the (unevaluated) products $S_{\mathbf{i}_{q}}=P_{i_{q}} \ldots P_{i_{2}} P_{i_{1}}$ of $q$ off-diagonal operators. Here we use the multiple index $\mathbf{i}_{q}=\left(i_{1}, \ldots, i_{q}\right)$ where each individual index $i_{j}$ ranges from 1 to $M$. In this notation, the empty sequence $S_{\mathbf{i}_{0}}$ corresponds to the identity operation. After some

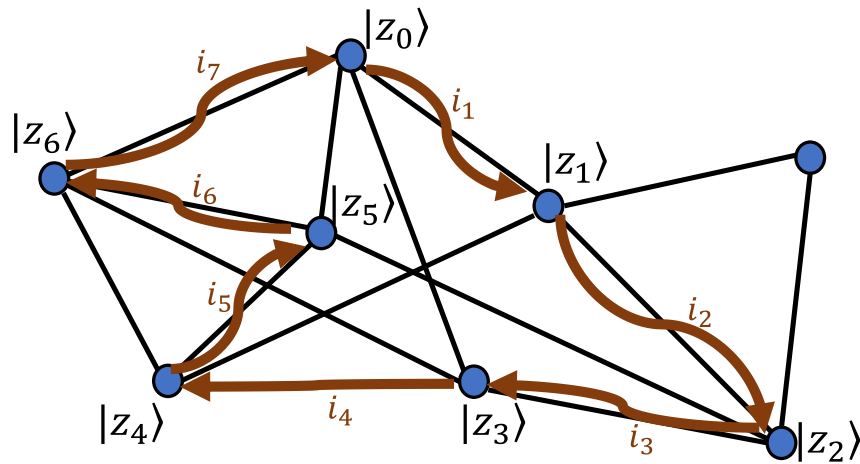

FIG. 1. Every summand $W_{\left(z, \mathbf{i}_{q}\right)}$ in the partition function decomposition is associated with a closed walk on the hypercube of basis states (a subgraph of which is shown here for illustration). The graph nodes (in blue) represent computational basis states and edges denote nonzero off-diagonal matrix elements. A walk on the graph (arrows in brown) is determined by the action of the permutation operators of the configuration, represented by the sequence $S_{\mathrm{i}_{q}}=P_{i_{q}} \cdots P_{i_{2}} P_{i_{1}}$, on the initial basis state $\left|z_{0}\right\rangle$. Every node $\left|z_{j}\right\rangle$ has an associated classical energy $E_{z_{j}}$.

algebra (the reader is referred to Ref. [9] for a full derivation), the partition function attains the form

$$
Z=\sum_{\{z\}} \sum_{q=0}^{\infty} \sum_{\mathbf{i}_{q}} D_{\left(z, \mathbf{i}_{q}\right)}\left\langle z\left|S_{\mathbf{i}_{q}}\right| z\right\rangle e^{-\beta\left[E_{z_{0}}, \ldots, E_{z q}\right]},
$$

where $\left\{S_{\mathbf{i}_{q}}\right\}$ is the set of all (unevaluated) products $P_{i_{q}} \ldots P_{i_{2}} P_{i_{1}}$ of size $q$ and $e^{-\beta\left[E_{z_{0}}, \ldots, E_{z q}\right]}$ is a divided difference of $f(\cdot)=e^{-\beta(\cdot)}$ (see Appendix A for an overview) with inputs $\left[E_{z_{0}}, \ldots E_{z_{q}}\right][22,23]$. The energies $E_{z_{i}}=\left\langle z_{i}\left|D_{0}\right| z_{i}\right\rangle$ (where $i=$ $0, \ldots, q)$ are the classical energies of the states $\left|z_{0}\right\rangle, \ldots,\left|z_{q}\right\rangle$, which are in turn obtained from the action of the ordered $P_{j}$ operators in the sequence $S_{\mathbf{i}_{q}}$ on $\left|z_{0}\right\rangle$, then on $\left|z_{1}\right\rangle$, and so forth. Explicitly, $\left|z_{0}\right\rangle=|z\rangle, P_{i_{1}}\left|z_{0}\right\rangle=\left|z_{1}\right\rangle, P_{i_{2}}\left|z_{1}\right\rangle=\left|z_{2}\right\rangle$, etc. The sequence of basis states $\left\{\left|z_{i}\right\rangle\right\}$ may be viewed as a closed walk [24] of length $q$ on the hypercube of basis states. See Fig. 1 for an illustration. The expression $\left\langle z\left|S_{\mathbf{i}_{q}}\right| z\right\rangle$ is 1 if $S_{\mathbf{i}_{q}}$ evaluates to the identity operator. Otherwise it is zero and can be removed from the sum. (Note that $\left|z_{j}\right\rangle=P_{i_{j}} \ldots P_{i_{2}} P_{i_{1}}|z\rangle$ in principle should be denoted $\left|z_{\left(i_{1}, \ldots, i_{j}\right)}\right\rangle$. We use a simplified notation so as not to overburden the notation.) Additionally, we denote

$$
D_{\left(z, \mathbf{i}_{q}\right)}=\prod_{j=1}^{q} d_{z_{j}}^{\left(\mathbf{i}_{j}\right)}
$$

where $d_{z_{j}}^{\left(\mathbf{i}_{j}\right)}=\left\langle z_{j}\left|D_{i_{j}}\right| z_{j}\right\rangle$ are off-diagonal elements of $H$.

The summands of the partition function decomposition are thus $D_{\left(z, \mathbf{i}_{q}\right)} e^{-\beta\left[E_{z 0}, \ldots, E_{z q}\right]}$. These can, in general, be complex valued, despite the partition function being real (the $d_{z_{j}}^{\left(\mathbf{i}_{j}\right)}$ can, in the general case, be complex). However, we note that for every configuration $\left(z, \mathbf{i}_{q}\right)$ there is a conjugate configuration $\left(z, \mathbf{i}_{q}^{\prime}\right)$, which produces the conjugate weight $W_{\left(z, \mathbf{i}_{q}^{\prime}\right)}=\bar{W}_{\left(z, \mathbf{i}_{q}\right)}$. Explicitly, for every closed walk $S_{\mathbf{i}_{q}}=P_{i_{q}} \ldots P_{i_{2}} P_{i_{1}}$ there is a conjugate walk in the reverse direction, whose operator sequence is $S_{\mathbf{i}_{q}}^{\dagger}=P_{i_{1}}^{-1} P_{i_{2}}^{-1} \ldots P_{i_{q}}^{-1}$ (see Fig. 2 for an illustration). The imaginary parts of the complex-valued summands 


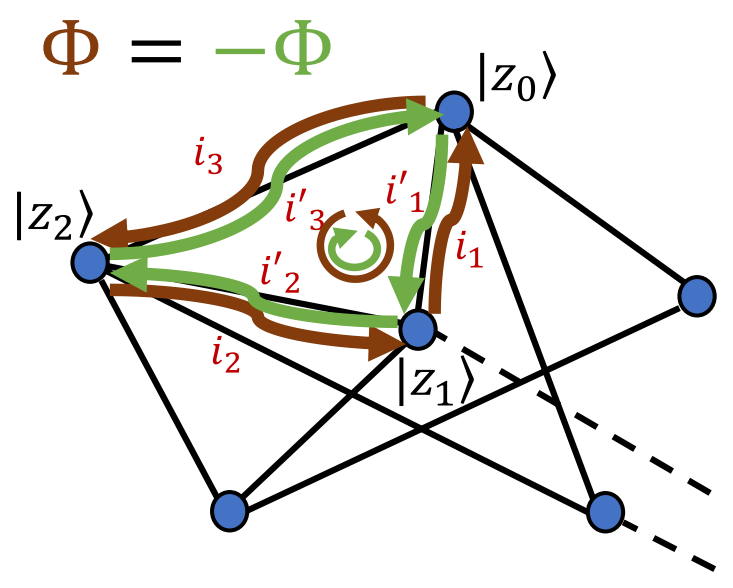

FIG. 2. Every closed walk (arrows in brown along edges of the graph) has a conjugate closed walk traversing the same path but in the reverse direction (arrows in green). The weight of a walk is the complex conjugate of the weight of its conjugate walk. This observation allows us to disregard the imaginary parts of these partition function summands which cancel out. The permutation operators that generate a closed walk are the inverses of the permutation operators that generate its conjugate and appear in reverse order.

therefore do not contribute to the partition function and may be disregarded altogether. We may therefore take

$$
W_{\left(z, \mathbf{i}_{q}\right)}=\operatorname{Re}\left[D_{\left(z, \mathbf{i}_{q}\right)}\right] e^{-\beta\left[E_{z_{0}}, \ldots, E_{z q}\right]}
$$

as the summands of the expansion.

We can now examine the condition for the positivity of the summands $W_{\left(z, \mathbf{i}_{q}\right)}$. First, we note that the term $e^{-\beta\left[E_{z_{0}}, \ldots, E_{z_{q}}\right]}$ is positive (negative) for even (odd) values of $q$, the length of the walk (see Appendix B for a short proof), and so the sign of a summand can be simplified to

$$
\operatorname{sgn}\left[W_{\left(z, \mathbf{i}_{q}\right)}\right]=\operatorname{sgn} \operatorname{Re}\left[\prod_{j=1}^{q}\left(-d_{z_{j}}^{\left(\mathbf{i}_{j}\right)}\right)\right],
$$

i.e., the partition function expansion will admit a negative weight if and only if there exists a closed walk on the hypercube of basis states along which $\operatorname{Re}\left[\prod_{j=1}^{q}\left(-d_{z_{j}}^{\left(\mathbf{i}_{j}\right)}\right)\right]<0$.

\section{From walks to cycles}

The expression derived above for the sign of a partition function summand can be given a more geometrical meaning if we write the off-diagonal matrix elements $d_{z_{j}}^{\left(\mathbf{i}_{j}\right)}$ in polar coordinates, i.e., $d_{z_{j}}^{\left(\mathbf{i}_{j}\right)}=-r_{z_{j}}^{\left(\mathbf{i}_{j}\right)} e^{-i \phi_{z_{j}}^{\left(\mathbf{i}_{j}\right)}}$ (note the extra minus sign introduced for notational convenience). This allows us to write the sign of a summand as

$$
\operatorname{sgn}\left[W_{\left(z, \mathbf{i}_{q}\right)}\right]=\operatorname{sgn} \operatorname{Re}\left[\prod_{j=1}^{q}\left(e^{-i \phi_{z j}^{\left(\mathbf{i}_{j}\right)}}\right)\right]=\operatorname{sgn} \cos \Phi_{\left(z, \mathbf{i}_{q}\right)},
$$

where we define

$$
\Phi_{\left(z, \mathbf{i}_{q}\right)}=\sum_{j=1}^{q} \phi_{z_{j}}^{\left(\mathbf{i}_{j}\right)}
$$

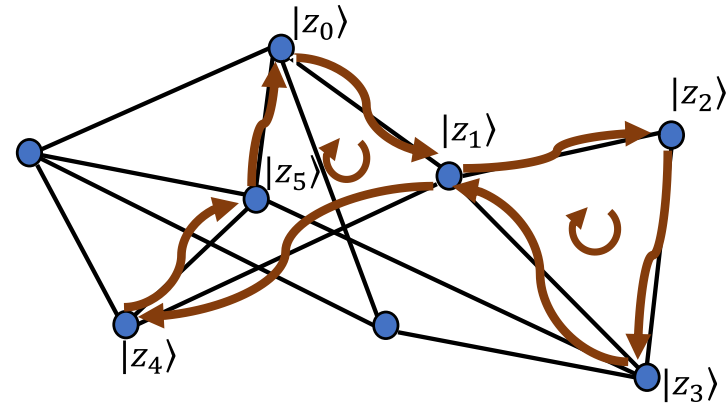

FIG. 3. Composite walks can be viewed as a concatenation of cycles (cyclic paths on a graph). Above, the closed walk $\left|z_{0}\right\rangle \rightarrow\left|z_{1}\right\rangle \rightarrow \cdots \rightarrow\left|z_{5}\right\rangle \rightarrow\left|z_{1}\right\rangle$ can be decomposed to two cycles: $\left|z_{1}\right\rangle \rightarrow\left|z_{2}\right\rangle \rightarrow\left|z_{3}\right\rangle \rightarrow\left|z_{1}\right\rangle$ and $\left|z_{0}\right\rangle \rightarrow\left|z_{1}\right\rangle \rightarrow\left|z_{4}\right\rangle \rightarrow\left|z_{5}\right\rangle \rightarrow$ $\left|z_{0}\right\rangle$. The overall phase associated with a closed walk is the sum of the phases associated with its constituent cycles.

as the overall geometric phase associated with the summand walk. For a Hamiltonian to be SPF, one must ensure that all closed walks have a positive $\cos \Phi_{\left(z, \mathbf{i}_{q}\right)}$.

The number of closed walks on the hypercube of basis states for any given Hamiltonian is infinite, even for finite graphs, since nodes can be revisited. Nonetheless, every closed walk may be viewed as the concatenation of distinct closed paths or cycles (equivalently, nonrepeating walks). Moreover, the phase of any given closed walk may be written as the sum of the phases of its constituent cycles (see Fig. 3 for an illustration).

Furthermore, long cycles may be viewed as the concatenation of smaller, basic, or induced (also referred to as chordless) cycles [25] that cannot in turn be decomposed to yet shorter cycles (see Fig. 4) and the overall phase associated with long cycles is the sum total of the phases of its constituent induced cycles.

\section{Geometric phases of the chordless cycles of SPF Hamiltonians}

Next, we answer the question of what values closed-walk phases can take to ensure that a given Hamiltonian has no sign problem. Let us consider an induced cycle with a total

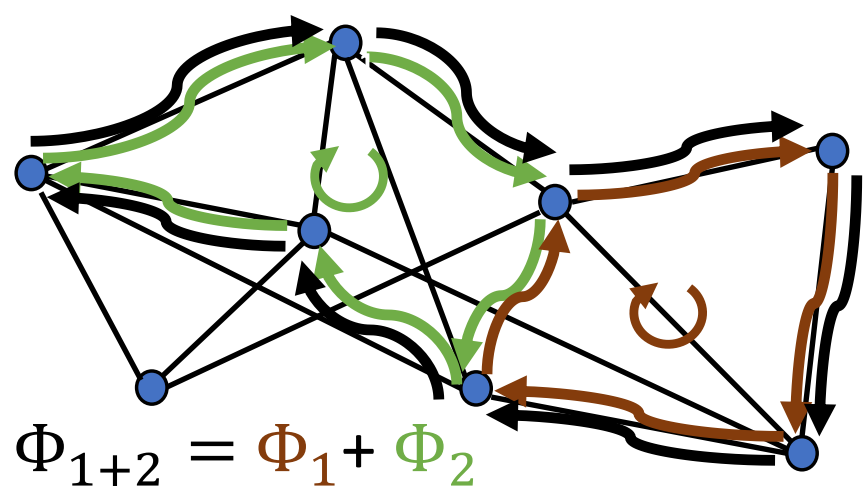

FIG. 4. The geometric phase associated with a composite cycle (black) is the total sum of the phases of its subcycles (brown and green). 
phase $\Phi$. As per Eq. (24), as long as $\cos \Phi \geqslant 0$, the weight associated with the cycle is positive [i.e., $\Phi \in(-\pi / 2, \pi / 2)$ modulo $2 \pi$ ]. However, since walks consisting of repeated concatenations of the original cycle are also legitimate walks, one must therefore also ensure that $\cos \Phi_{m}=\cos m \Phi \geqslant 0$ for every natural number $m$. For this much stronger condition to hold, the induced-cycle phase must be $\Phi=0$ modulo $2 \pi$ (see Appendix C), i.e., the cycle must have a vanishing geometric phase (VGP).

Having observed that the chordless cycles of a Hamiltonian must have VGPs for their associated weights to be positive (we call these VGP Hamiltonians), it is straightforward to generalize the condition to include longer cycles and, in fact, all closed walks: If all cycles of a Hamiltonian have vanishing phases, then all closed walks, which are concatenations of cycles, have vanishing phases as well. We thus conclude that for general Hamiltonians to have no sign problem in a given basis, the geometric phases of the chordless cycles of their Hamiltonian must be zero modulo $2 \pi$. This condition is necessary and sufficient (although the efficiency with which it can be checked for different classes of Hamiltonians, particularly bounded locality ones, is yet to be determined).

In the special case where all $d_{z_{j}}^{\left(\mathbf{i}_{j}\right)}$, which correspond to offdiagonal Hamiltonian elements, are negative and hence have zero phase $\phi_{z_{j}}^{\left(\mathbf{i}_{j}\right)}=0$, we obtain $\Phi_{\left(z, \mathbf{i}_{q}\right)}=0$ for every closed walk. This in turn implies that the weights associated with all walks are positive and the Hamiltonian will be SPF. This is the case of stoquastic Hamiltonians.

To illustrate the difference between stoquasticity and VGP, in the next section we introduce a class of Hamiltonians that are nonstoquastic yet VGP, making them QMC-simulable SPF Hamiltonians.

\section{NONSTOQUASTIC SIGN-PROBLEM-FREE HAMILTONIANS}

Let us consider a general $N$-by- $N$ stoquastic Hamiltonian $H^{\text {(stoq) }}$, i.e., a general symmetric matrix with nonpositive offdiagonal elements (explicitly, $H_{i j}^{(\text {stoq) }} \leqslant 0$ for all $i \neq j$ where $i, j \in\{1, \ldots, N\})$. We next define

$$
H^{(\mathrm{VGP})}=e^{i \Theta} H^{(\mathrm{stoq})} e^{-i \Theta},
$$

where $\Theta=\operatorname{diag}\left\{\theta_{1}, \ldots, \theta_{N}\right\}$ is a diagonal matrix of phases. The off-diagonal elements of $H^{(\mathrm{VGP})}$ are

$$
H_{i j}^{(\mathrm{VGP})}=H_{i j}^{(\mathrm{stoq})} e^{i\left(\theta_{i}-\theta_{j}\right)},
$$

and so $H^{(\mathrm{VGP})}$ will generally be nonstoquastic. Nonetheless, it is straightforward to show that $H^{(\mathrm{VGP})}$ above is VGP. This is because for any closed walk, i.e., a sequence of off-diagonal elements, we have

$$
\begin{aligned}
& \left(-H_{i j}^{(\mathrm{VGP})}\right)\left(-H_{j k}^{(\mathrm{VGP})}\right) \cdots\left(-H_{m l}^{(\mathrm{VGP})}\right)\left(-H_{l i}^{(\mathrm{VGP})}\right) \\
& \quad=\left(-H_{i j}^{\text {(stoq) }} e^{i\left(\theta_{i}-\theta_{j}\right)}\right)\left(-H_{j k}^{\text {(stoq })} e^{i\left(\theta_{j}-\theta_{k}\right)}\right) \cdots \\
& \quad=\left(-H_{i j}^{\text {(stoq) }}\right)\left(-H_{j k}^{\text {(stoq) }}\right) \cdots\left(-H_{m l}^{\text {(stoq) }}\right)\left(-H_{l i}^{\text {(stoq) }}\right) \geqslant 0 .
\end{aligned}
$$

The converse is also true: If a Hamiltonian is VGP, it can always be unitarily transformed to stoquastic form via a rotation by a phase matrix. To show this, consider a VGP Hamiltonian whose off-diagonal elements are written in polar form $H_{i j}^{(\mathrm{VGP})}=-r_{i j} e^{i \phi_{i j}}$. Its phases obey $\phi_{j i}=-\phi_{i j}$ (Hermiticity) and $\left(\phi_{i j}+\phi_{j k}+\cdots+\phi_{m l}+\phi_{l i}\right)=0$ modulo $2 \pi$ (VGP) provided that all of $r_{i j}, r_{j k}, \ldots, r_{m l}, r_{l i}>0$. We will prove that there is always a rotation matrix $e^{i \Theta}$ that can rotate $H^{(\mathrm{VGP})}$ to stoquastic form; that is, we will show that there is always a choice $\left\{\theta_{1}, \ldots, \theta_{N}\right\}$ such that $\phi_{i j}+\theta_{i}-\theta_{j}=0$ modulo $2 \pi$ for all $i \neq j$. To do that, we will prove that there are at most $N-1$ independent phases, which if cured imply the curing of all other phases, owing to the VGP condition. Since there are $N-1$ independent choices for $\theta_{i}-\theta_{j}$, we can choose $\theta_{i}-\theta_{j}=-\phi_{i j}$ for all independent phases, thereby curing all phases.

To show that there are at most $N-1$ independent phases, let us assume for simplicity that all off-diagonal elements are nonzero, that is $r_{i j}>0$ for all $i \neq j$ (a proof for the general case, in which this condition is absent, is given in Appendix D). It is easy to show that the $N-1$ phases $\phi_{12}, \ldots, \phi_{1 N}$ determine all other phases. From Hermiticity, we have $\phi_{m 1}=-\phi_{1 m}$ for all $m=2, \ldots, N$. Next, consider the phases of all three-cycles $\left(-H_{1 n}^{(\mathrm{VGP})}\right)\left(-H_{n m}^{(\mathrm{VGP})}\right)\left(-H_{m 1}^{(\mathrm{VGP})}\right)$. From VGP, we know that $\phi_{1 n}+\phi_{n m}+\phi_{m 1}=0$ for all $n, m \neq$ 1 , which in turn implies that all $\phi_{n m}$ are defined modulo $2 \pi$. If all $\phi_{1 m}$ are cured after rotation, then so will all $\phi_{m 1}$, and due to VGP, the same is true for all $\phi_{n m}$.

Note that the curing transformation $e^{i \Theta}$ is a nonlocal one in general, and finding it requires solving a linear set of (up to) $N-1$ equations. The efficiency with which such a transformation can be found or applied for bounded locality or other classes of Hamiltonians is not determined at this point. As previously noted, however, there is no actual need for curing nonstoquastic VGP Hamiltonians because they are $\mathrm{SPF}$ to begin with.

Because the VGP condition is both necessary and sufficient for QMC simulability, we can give a complete characterization for all SPF Hamiltonians: These must be of the form $e^{i \Theta} H^{(\text {stoq) }} e^{-i \Theta}$, i.e., SPF Hamiltonians are diagonally rotated stoquastic Hamiltonians.

At this point, it would be instructive to illustrate the distinction between stoquasticity and the VGP property by examining a specific model. One interesting example, which was recently introduced in Ref. [18] as a one-dimensional model that is nonstoquastic and yet does not produce a sign problem, is

$$
H=\sum_{i} X_{i}-\frac{1}{2} \sum_{i<j}\left(X_{i} X_{j}-Y_{i} Y_{j}\right) .
$$

In this model, the $X_{i}$ operators produce the nonstoquasticity, yielding positive-valued off-diagonal matrix elements. In PMR form, the Hamiltonian can be rewritten as

$$
H=\sum_{i} X_{i}-\frac{1}{2} \sum_{i<j}\left(1_{i j}+Z_{i} Z_{j}\right) X_{i} X_{j} .
$$

We therefore identify two types of off-diagonal permutations: $P_{i}=X_{i}$ with $D_{i}=1_{i}$ as their accompanying diagonal matrices and $P_{i j}=X_{i} X_{j}$ with which $D_{i j}=-\frac{1}{2}\left(1_{i j}+Z_{i} Z_{j}\right)=$ $\operatorname{diag}\{-1,0,0,-1\}$ are associated. 


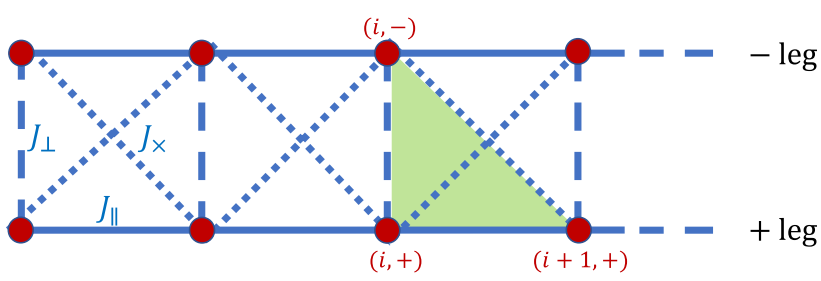

FIG. 5. The frustrated Heisenberg two-leg spin 1/2 ladder. The Heisenberg-type interactions between the spins (vertices in red) are denoted by the edges connecting the vertices. The coupling strengths associated with the solid, dashed, and dotted edges are $J_{\|}$(horizontal), $J_{\perp}$ (vertical), and $J_{\times}$(diagonal), respectively. The edges that make up the contour of the shaded area (in green), connecting the spins $(i,+),(i+1,+)$, and $(i,-)$ are an example of a triplet of edges that may yield negative-weight closed walks.

The chordless cycles of this model are formed by sequences of operators of the form $P_{i j} P_{j} P_{i}$ (or permutations thereof) which generate cycles of the form $|z\rangle \rightarrow\left|z_{i}\right\rangle \rightarrow$ $\left|z_{i j}\right\rangle \rightarrow|z\rangle$ where $|z\rangle$ is any starting node and $\left|z_{i}\right\rangle\left(\left|z_{i j}\right\rangle\right)$ stands for the basis state $|z\rangle$ with its $i$ th spin (ith and $j$ th spins) flipped. The signs of the weights associated with these cycles are determined by the sign of the product $\left(-d_{z_{i}}^{(i)}\right)\left(-d_{z_{i j}}^{(j)}\right)\left(-d_{z}^{(i j)}\right)$ [as per Eq. (5)] where $d_{z_{i}}^{(i)}=\left\langle z_{i}\left|D_{i}\right| z_{i}\right\rangle$, $d_{z_{i j}}^{(j)}=\left\langle z_{i j}\left|D_{j}\right| z_{i j}\right\rangle$ and $d_{z}^{(i j)}=\left\langle z\left|D_{i j}\right| z\right\rangle$. One can easily check that for any chosen starting node $|z\rangle$, we have $d_{z_{i}}^{(i)}=d_{z_{i j}}^{(j)}=1$ (a $\pi$ phase) and $d_{z}^{(i j)}=-1$ (a zero phase) or 0 depending on the state $z$. In the case where $d_{z}^{(i j)}=0$, the overall weight is zero. In the $d_{z}^{(i j)}=-1$ case, the overall sign of the weight is necessarily positive (the total phase is zero), implying an SPF system, despite the apparent nonstoquasticity of the model.

One can further rotate the model to an explicitly stoquastic representation by applying the diagonal unitary $U_{Z}=\bigotimes Z_{i}$. This transformation has the effect $X_{i} \rightarrow-X_{i}$ and $Y_{i} \rightarrow-Y_{i}$, reversing the sign of the one-body $X_{i}$ terms as desired while leaving the two-body terms unchanged.

Another example that the sign problem of which is worth formally deriving is the frustrated Heisenberg two-leg spin $1 / 2$ ladder with $L$ rungs (this model and its sign problem were studied numerically in Ref. [26]). The Hamiltonian of the model is

$$
H=J_{\perp} \sum_{i} \vec{S}_{i}^{+} \vec{S}_{i}^{-}+J_{\|} \sum_{i, m= \pm} \vec{S}_{i}^{m} \vec{S}_{i+1}^{m}+J_{\times} \sum_{i, m= \pm} \vec{S}_{i}^{m} \vec{S}_{i+1}^{-m},
$$

where $i$ is the rung index, $m= \pm$ denotes the two legs of the ladder, and the parameters, $J_{\perp}, J_{\|}$, and $J_{\times}$are the horizontal (ladder axis), vertical (connecting the two legs), and diagonal coupling strengths of the Heisenberg interactions between connected spins. See Fig. 5 for an illustration.

In PMR form, the Hamiltonian reads

$$
\begin{aligned}
H= & D_{0}+\sum_{i} D_{i}^{\perp} X_{i}^{+} X_{i}^{-}+\sum_{i, m= \pm} D_{i, m}^{\|} X_{i}^{m} X_{i+1}^{m} \\
& +\sum_{i, m= \pm} D_{i, m}^{\times} X_{i}^{m} X_{i+1}^{-m},
\end{aligned}
$$

where the diagonal component $D_{0}$ is identified as

$$
D_{0}=J_{\perp} \sum_{i} Z_{i}^{+} Z_{i}^{-}+J_{\|} \sum_{i, m= \pm} Z_{i}^{m} Z_{i+1}^{m}+J_{\times} \sum_{i, m= \pm} Z_{i}^{m} Z_{i+1}^{-m} .
$$

In addition, we have denoted $D_{i}^{\perp}=J_{\perp}\left(1-Z_{i}^{+} Z_{i}^{-}\right), D_{i, m}^{\|}=$ $J_{\|}\left(1-Z_{i}^{m} Z_{i+1}^{m}\right)$ and $D_{i, m}^{\times}=J_{\times}\left(1-Z_{i}^{m} Z_{i+1}^{-m}\right)$. The permutation operators in this representation are, correspondingly, $P_{i}^{\perp}=$ $X_{i}^{+} X_{i}^{-}, P_{i, m}^{\|}=X_{i}^{m} X_{i+1}^{m}$ and $P_{i, m}^{\times}=X_{i}^{m} X_{i+1}^{-m}$.

For the above model, three-cycles of permutation operators that evaluate to the identity are of the form $P_{i, m}^{\|}$. $P_{i,-m}^{\times} \cdot P_{i}^{\perp}$ or permutations thereof. For positive-valued $J_{\|}$, $J_{\perp}$, and $J_{\times}$, the matrix elements of the associated $\left(-D_{i, m}^{\|}\right)$, $\left(-D_{i, m}^{\times}\right)$and $\left(-D_{i}^{\perp}\right)$ matrices are either negative or zero and so products thereof, which determine the signs of the weights generated from such operator sequences, are similarly either negative or zero, depending on the configuration of the spins sitting at the vertices $(i, m),(i,-m)$ and $(i+$ $1, m$ ) (see Fig. 5). For basis states such as $|z\rangle=\mid \ldots \uparrow \downarrow \uparrow$ $\ldots\rangle$, the above sequence of permutation operators generates the closed walk $|\ldots \uparrow \downarrow \uparrow \ldots\rangle \rightarrow|\ldots \downarrow \uparrow \uparrow \ldots\rangle \rightarrow \mid \ldots \uparrow \uparrow \downarrow$ $\ldots\rangle \rightarrow|\ldots \uparrow \downarrow \uparrow \ldots\rangle$ which in turn produces a strictly negative weight and hence a sign problem.

\section{QMC SIMULATIONS WITHOUT STOQUASTIZATION}

The VGP Hamiltonians discussed in the previous section illustrate an additional point of practical significance to the QMC simulation of (truly) sign-problematic systems that admit negative terms in the partition function decomposition.

To demonstrate this point, let us first briefly overview the impact of the sign problem on the efficiency of QMC simulations. The reason the sign problem is indeed a serious impediment to QMC algorithms is encompassed in the fact that the computational efficiency of QMC simulations hinges on the algorithm's ability to sample the configurations $\mathcal{C}$ [in our case, $\mathcal{C}$ are labeled by pairs $\left.\left(z, \mathbf{i}_{q}\right)\right]$, as obtained from the partition function decomposition, according to their relative weights, $p_{\mathcal{C}}=W_{\mathcal{C}} / Z$.

A necessary condition for proper importance-based sampling (or importance sampling for short) is that all weights $W_{\mathcal{C}}$ are positive (or nonnegative), i.e., that $p_{\mathcal{C}}$ can be interpreted as a genuine probability distribution over configurations. Whenever $p_{\mathcal{C}}$ is a proper probability distribution, the thermal average $\langle A\rangle$ of any physical observable $A$ can be written as

$$
\langle A\rangle=\frac{\operatorname{Tr}\left[A e^{-\beta H}\right]}{Z}=\frac{\sum_{\mathcal{C}} A_{\mathcal{C}} W_{\mathcal{C}}}{\sum_{\mathcal{C}} W_{\mathcal{C}}}=\sum_{\mathcal{C}} A_{\mathcal{C}} p_{\mathcal{C}} .
$$

Since an explicit summation over all terms in the sum above is, in general, prohibitive due to the generally exponential number of summands, QMC importance sampling estimates the sum using a Monte Carlo estimator, defined as

$$
\langle\tilde{A}\rangle_{p} \approx \frac{1}{N_{\mathrm{s}}} \sum_{i=1}^{N_{\mathrm{s}}} A_{\mathcal{C}_{i}}
$$

where the configurations $\mathcal{C}_{i}$ (of which there are $N_{\mathrm{s}}$ ) are randomly sampled in proportion to their probability $p_{\mathcal{C}_{i}}$ [27]. If 
the number of important configurations is relatively small, then estimation via importance sampling will converge considerably faster than with straightforward unbiased sampling of the terms (or an actual summation thereof) and oftentimes exponentially more so. (It is important to point out though that in neither case polynomial-time convergence of the algorithm is guaranteed.)

Importance sampling will not be possible if a subset of the configurations is assigned negative weights, as in this case $p_{\mathcal{C}}$ is no longer a bona fide probability distribution. The common workaround in QMC for the occurrence of negative weights is to draw samples from a different distribution,

$$
\tilde{p}_{\mathcal{C}}=\frac{\widetilde{W}_{\mathcal{C}}}{\sum_{\mathcal{C}} \widetilde{W}_{\mathcal{C}}}
$$

that is nonnegative everywhere $[10,28]$. Then, the estimator of $A$ becomes

$$
\langle\tilde{A}\rangle_{\tilde{p}} \approx \frac{1}{N_{\mathrm{s}}} \sum_{i=1}^{N_{\mathrm{s}}} A_{\mathcal{C}_{i}} \frac{p_{\mathcal{C}_{i}}}{\tilde{p}_{\mathcal{C}_{i}}},
$$

and thermal averages can be written as

$$
\langle A\rangle=\frac{\sum_{\mathcal{C}} A_{\mathcal{C}}\left(W_{\mathcal{C}} / \widetilde{W}_{\mathcal{C}}\right) \widetilde{W}_{\mathcal{C}}}{\sum_{\mathcal{C}}\left(W_{\mathcal{C}} / \widetilde{W}_{\mathcal{C}}\right) \widetilde{W}_{\mathcal{C}}}=\frac{\langle A \operatorname{sgn}\rangle_{\widetilde{W}}}{\langle\operatorname{sgn}\rangle_{\widetilde{W}}}
$$

where sgn above stands for the ratio $W_{\mathcal{C}} / \widetilde{W}_{\mathcal{C}}$ and $\langle\cdot\rangle_{\widetilde{W}}$ denotes averaging with respect to the genuine (unnormalized) nonnegative distribution $\widetilde{W}$. The above thermal average is therefore the ratio of two other thermal averages, both of which sampled according to $\widetilde{W}$.

The denominator $\langle\operatorname{sgn}\rangle_{\widetilde{W}}$ is often referred to as the weighted sign or average sign and is simply the ratio of the sum of original weights $W_{\mathcal{C}}$ (i.e., the partition function of the system in question) to the sum of the nonnegative weights chosen for the simulation $\widetilde{W}_{\mathcal{C}}[8,10]$, namely,

$$
\langle\operatorname{sgn}\rangle_{\widetilde{W}}=\frac{\sum_{\mathcal{C}} W_{\mathcal{C}}}{\sum_{\mathcal{C}} \widetilde{W}_{\mathcal{C}}} .
$$

The average sign often serves as a continuous measure for the simulability of the model. For SPF models where all weights are positive, the $\widetilde{W}_{\mathcal{C}}$ distribution coincides with $W_{\mathcal{C}}$ and $\langle\operatorname{sgn}\rangle_{\widetilde{W}}=1$. For non-SPF models, on the other hand, $\langle\operatorname{sgn}\rangle_{\widetilde{W}}$ will be strictly smaller than 1 , approaching zero from above the more dominant the negative weights are. In these cases, the summands in the numerator will fluctuate rapidly around zero and so estimation of thermal averages will require an inordinately large number of samples [10,29]. The average sign is therefore a measure of how adverse the sign problem is in a QMC simulation with QMC convergence times inversely proportional to the value of the weighted sign.

Of course, the value of the weighted sign $\langle\operatorname{sgn}\rangle_{\widetilde{W}}$ depends on the distribution $\widetilde{W}$. A common choice for the weights $\widetilde{W}$ are the QMC weights associated with the stoquasticized version of the Hamiltonian (see, e.g., Refs. [10,30]). Explicitly, the weights are taken to be those generated from the decomposition of the partition function of $H^{\text {(stoq) }}$, the stoquasticized version of the Hamiltonian obtained by $H_{i j}^{\text {(stoq) }}=-\left|H_{i j}\right|$ for all $i \neq j$, which are guaranteed to be positive (this choice is sometimes referred to as bosonization). In our notation, the positive stoquasticized weights are given by

$$
W_{\left(z, \mathbf{i}_{q}\right)}^{(\text {stoq }}=\left|D_{\left(z, \mathbf{i}_{q}\right)} e^{-\beta\left[E_{z_{0}}, \ldots, E_{z q}\right]}\right| .
$$

These are immediately obtained upon switching $d_{z_{j}}^{\left(\mathbf{i}_{j}\right)} \rightarrow$ $-\left|d_{z_{j}}^{\left(\mathbf{i}_{j}\right)}\right|$. For this choice, the weighted sign becomes

$$
\langle\operatorname{sgn}\rangle_{\text {stoq }}=\frac{\sum_{\left(z, \mathbf{i}_{q}\right)} W_{\left(z, \mathbf{i}_{q}\right)}}{\sum_{\left(z, \mathbf{i}_{q}\right)} W_{\left(z, \mathbf{i}_{q}\right)}^{(\text {stoq })}} .
$$

We argue though that the above choice is, in general, not optimal and that a more appropriate choice of positive weights is to place the absolute value on the cosine of the total phase of each walk, namely,

$$
W_{\left(z, \mathbf{i}_{q}\right)}^{(\mathrm{ABS})}=\left|W_{\left(z, \mathbf{i}_{q}\right)}\right|=\left|\cos \Phi_{\left(z, \mathbf{i}_{q}\right)}\right| W_{\left(z, \mathbf{i}_{q}\right)}^{(\mathrm{stoq})} .
$$

This choice leads to a weighted sign of

$$
\langle\operatorname{sgn}\rangle_{\mathrm{ABS}}=\frac{\sum_{\left(z, \mathbf{i}_{q}\right)} W_{\left(z, \mathbf{i}_{q}\right)}}{\sum_{\left(z, \mathbf{i}_{q}\right)} W_{\left(z, \mathbf{i}_{q}\right)}^{(\mathrm{ABS})}}=\frac{\sum_{\left(z, \mathbf{i}_{q}\right)} W_{\left(z, \mathbf{i}_{q}\right)}}{\sum_{\left(z, \mathbf{i}_{q}\right)}\left|\cos \Phi_{\left(z, \mathbf{i}_{q}\right)}\right| W_{\left(z, \mathbf{i}_{q}\right)}^{(\text {stoq })}},
$$

from which it is clear that

$$
\langle\operatorname{sgn}\rangle_{\mathrm{ABS}} \geqslant\langle\operatorname{sgn}\rangle_{\mathrm{stoq}},
$$

with equality only when $\langle\operatorname{sgn}\rangle_{\mathrm{ABS}}=1$, i.e., for SPF Hamiltonians. In all other cases, where $\langle\operatorname{sgn}\rangle_{\mathrm{ABS}}<1$, the inequality is a strict one and the sign problem becomes provably less severe for the latter alternative. In fact, in the low-temperature limit, we expect $\lim _{\beta \rightarrow \infty}\langle\text { sgn }\rangle_{\mathrm{ABS}} /\langle\operatorname{sgn}\rangle_{\text {stoq }}=\infty$ as both quantities decay exponentially fast to zero as a function of $\beta[8,10,18]$ but at different rates.

\section{SUMMARY AND CONCLUSIONS}

We gave a necessary and sufficient condition for the QMC simulability of Hamiltonians in a fixed basis. We found that if and only if all the geometric phases of the chordless cycles of the weighted graph whose adjacency matrix is the Hamiltonian are zero (modulo $2 \pi$ ), the simulation will be SPF, a condition we call VGP. To further distinguish simulability from nonstoquasticity, we presented a construction for all (generally) nonstoquastic yet efficiently simulable (equivalently, SPF) Hamiltonians.

We also showed that simulating sign-problematic Hamiltonians by choosing the weights to be the summands of the partition function decomposition of the analogous stoquasticized Hamiltonian, i.e., the stoquastic analog of the Hamiltonian, is a suboptimal choice in general. We provided a more suitable choice for said weights in terms of the absolute values of the cosines of the geometric phases of the simulated Hamiltonian, the weighted sign due to which is bounded from below by the weighted sign generated by the stoquastic choice. We can therefore expect that the choice of positive weights proposed here will allow for more efficient QMC simulations of sign-problematic quantum many-body models and, in turn, will enable the study of considerably larger systems than possible by stoquastization.

Our study advocates the examination of the conditions under which Hamiltonians can be unitarily transformed to 
VGP form, rather than to the less relevant stoquastic form, which is the common practice today for curing the sign problem (see, e.g., Refs. [14-18]). Especially worth mentioning in this context are recent results that have shown that for certain classes of Hamiltonians, curing nonstoquasticity, i.e., finding unitary transformations that transform the Hamiltonian to stoquastic form, is an intractable (NP-hard) task. The fact that nonstoquasticity does not imply nonsimulability suggests that demanding the curing of nonstoquasticity is, in general, too excessive and that the weaker condition of seeking transformations to VGP form are more appropriate. Moreover, our results explain why the application of unitary rotations that minimize the nonstoquasticity of Hamiltonians do not necessarily make them less sign-problematic (i.e., do not necessarily reduce their average sign) [18].

Developing a true understanding of the nature of the QMC sign problem stands to have implications across all branches of the physical sciences and is crucial to the potential resolution of the problem. We hope that this paper will provide a useful framework for making progress in this context.

Additional directions of research that we believe are worth pursuing are studying the extent to which the concept of VGP is also relevant for results pertaining to the use of stoquasticity in complexity theory [1-4] and in other domains, specifically in determining the positivity of Hamiltonian ground states [31] and the bounding of their spectral gaps [32]. In Ref. [32], for example, a similar generalization of stoquasticity was proposed in a different context.

While the VGP condition is the appropriate one to check when determining the existence of a sign problem, it is yet to be determined whether it is a more useful concept to work with than stoquasticity. Specifically, whether the complexity of verifying that a given Hamiltonian is VGP is different than the complexity associated with verifying that it is stoquastic is still an open question.

The question of whether all the results pertaining to stoquasticity hold for VGP as well is an interesting one. In the same context, another relevant question is whether diagonally rotating a bounded-locality VGP Hamiltonian to explicitly stoquastic form can always be done efficiently. An affirmative answer may serve to further cement the relation between stoquasticty and VGP. A negative answer may establish a fundamental distinction between stoquasticity and VGP, which would in turn make the identification of QMC-simulable systems more difficult and previous results pertaining to simulability by curing nonstoquasticity less impactful [14-18]. We leave these for future work.

\section{ACKNOWLEDGMENTS}

We thank Elizabeth Crosson, Michael Jarret, and Milad Marvian for valuable comments and discussions. The research is based upon work (partially) supported by the Office of the Director of National Intelligence (ODNI), Intelligence Advanced Research Projects Activity (IARPA) and the Defense Advanced Research Projects Agency (DARPA), via the U.S. Army Research Office Contract No. W911NF-17-C-0050. The views and conclusions contained herein are those of the authors and should not be interpreted as necessarily representing the official policies or endorsements, either expressed or implied, of the ODNI, IARPA, DARPA, or the U.S. Government. The U.S. Government is authorized to reproduce and distribute reprints for governmental purposes notwithstanding any copyright annotation thereon.

\section{APPENDIX A: NOTES ON DIVIDED DIFFERENCES}

We provide below a brief summary of the concept of divided differences, which is a recursive division process. This method is typically encountered when calculating the coefficients in the interpolation polynomial in the Newton form.

The divided differences [22,23] of a function $F(\cdot)$ is defined as

$$
F\left[x_{0}, \ldots, x_{q}\right] \equiv \sum_{j=0}^{q} \frac{F\left(x_{j}\right)}{\prod_{k \neq j}\left(x_{j}-x_{k}\right)}
$$

with respect to the list of real-valued input variables $\left[x_{0}, \ldots, x_{q}\right]$. The above expression is ill-defined if some of the inputs have repeated values, in which case one must resort to a limiting process. For instance, in the case where $x_{0}=x_{1}=$ $\cdots=x_{q}=x$, the definition of divided differences reduces to

$$
F\left[x_{0}, \ldots, x_{q}\right]=\frac{F^{(q)}(x)}{q !},
$$

where $F^{(n)}(\cdot)$ stands for the $n$th derivative of $F(\cdot)$. Divided differences can alternatively be defined via the recursion relations

$$
F\left[x_{i}, \ldots, x_{i+j}\right]=\frac{F\left[x_{i+1}, \ldots, x_{i+j}\right]-F\left[x_{i}, \ldots, x_{i+j-1}\right]}{x_{i+j}-x_{i}},
$$

with $i \in\{0, \ldots, q-j\}, j \in\{1, \ldots, q\}$ with the initial conditions

$$
F\left[x_{i}\right]=F\left(x_{i}\right), \quad i \in\{0, \ldots, q\} \quad \forall i .
$$

A function of divided differences can be defined in terms of its Taylor expansion. In the case where $F(x)=e^{-\beta x}$, we have

$$
e^{-\beta\left[x_{0}, \ldots, x_{q}\right]}=\sum_{n=0}^{\infty} \frac{(-\beta)^{n}\left[x_{0}, \ldots, x_{q}\right]^{n}}{n !} .
$$

\section{APPENDIX B: SIGN OF $e^{-\beta\left[E_{0}, \ldots, E_{q}\right]}$}

We note that $e^{\left[x_{0}, \ldots, x_{q}\right]}$ is positive for any set of inputs $x_{0}, \ldots, x_{q}[33,34]$. Setting $x_{j}=-\beta E_{j}$ for $j=0, \ldots, q$ yields $e^{\left[-\beta E_{0}, \ldots,-\beta E_{q}\right]}>0$. We next prove that

$$
e^{\left[-\beta E_{0}, \ldots,-\beta E_{q}\right]}=(-\beta)^{q} e^{-\beta\left[E_{0}, \ldots, E_{q}\right]} .
$$

This immediately follows from the definition of divided differences. Explicitly,

$$
\begin{aligned}
e^{\left[-\beta E_{0}, \ldots,-\beta E_{q}\right]} & =\sum_{j} \frac{e^{-\beta E_{j}}}{\prod_{k \neq j}\left(E_{j}-E_{k}\right)} \\
& =(-\beta)^{q} \sum_{j} \frac{e^{-\beta E_{j}}}{\prod_{k \neq j}\left(-\beta E_{j}+\beta E_{k}\right)} \\
& =(-\beta)^{q} e^{-\beta\left[E_{0}, \ldots, E_{q}\right]} .
\end{aligned}
$$


It follows then that

$$
\operatorname{sgn} e^{-\beta\left[E_{0}, \ldots, E_{q}\right]}=\operatorname{sgn}(-1)^{q} .
$$

\section{APPENDIX C: POSITIVITY OF $\cos m x$}

We show that the only $x \in[0,2 \pi)$ for which $\cos m x \geqslant 0$ for every natural number $m$ is $x=0$. Plugging $x=0$, we obtain $\cos 0=1>0$ for every $m$. Next we show that for any $x \in(0,2 \pi)$ there is an $m$ such that $\cos m x<0$. We break down the statement to three cases: (i) If $x$ is in the interval $(\pi / 2,3 \pi / 2)$, then $\cos x$ is already negative. (ii) If $x \in(0, \pi / 2]$, then consider the smallest $m$ for which $m x>$ $\pi / 2$. We have $(m-1) x \leqslant \pi / 2$ and $m x=(m-1) x+x>$ $\pi / 2$. Since $(m-1) x \leqslant \pi / 2$ and $0<x \leqslant \pi / 2$, then $m x$ is necessarily in $(\pi / 2, \pi]$ in which case $\cos m x<0$. (iii) If $x \in[3 \pi / 2,2 \pi)$, then consider the smallest $m$ for which ( $m x$ $\bmod 2 \pi)<3 \pi / 2$. Since $[(m-1) x \bmod 2 \pi] \geqslant 3 \pi / 2$ and $3 \pi / 2 \leqslant x<2 \pi$, then $m x$ is necessarily in $[\pi, 3 \pi / 2)$ and so $\cos m x<0$.

\section{APPENDIX D: CASTING VGP HAMILTONIANS IN STOQUASTIC FORM VIA DIAGONAL UNIATRIES}

Here, we prove that a Hamiltonian, $H^{(\mathrm{VGP})}$, obeying

$$
\left(-H_{i j}^{(\mathrm{VGP})}\right)\left(-H_{j k}^{(\mathrm{VGP})}\right) \cdots\left(-H_{m l}^{(\mathrm{VGP})}\right)\left(-H_{l i}^{(\mathrm{VGP})}\right) \geqslant 0
$$

for every set of indices $i \neq j \neq k \ldots m \neq l$, also referred to as a VGP Hamiltonian, can always be unitarily transformed to stoquastic form via a rotation by a phase matrix $e^{i \Theta}$ where $\Theta=\operatorname{diag}\left\{\theta_{1}, \ldots, \theta_{N}\right\}$ is a diagonal matrix of phases.

We first rewrite the Hamiltonian's off-diagonal elements in polar form, $H_{i j}^{(\mathrm{VGP})}=-r_{i j} e^{i \phi_{i j}}$. Its phases obey $\phi_{j i}=-\phi_{i j}$ (Hermiticity) and $\left(\phi_{i j}+\phi_{j k}+\cdots+\phi_{m l}+\phi_{l i}\right)=0$ modulo $2 \pi$ provided that all of $r_{i j}, r_{j k}, \ldots, r_{m l}, r_{l i}>0$ (VGP condition).

We will prove that there is always a rotation matrix $e^{i \Theta}$ that can rotate $H^{\text {(VGP) }}$ to stoquastic form; that is, we will show that there is always a choice $\left\{\theta_{1}, \ldots, \theta_{N}\right\}$ such that $\phi_{i j}+\theta_{i}-\theta_{j}=$ 0 modulo $2 \pi$ for all $i \neq j$.

Let us first show that $H^{(\mathrm{VGP})}$ can be partitioned to a finite set of noninteracting lower-dimensional VGP Hamiltonians. We do so by showing that the matrix elements $H_{i j}^{(\mathrm{VGP})}$ can be partitioned to equivalence classes defined via the equivalence relation of reachability as follows. An ordered pair of offdiagonal matrix elements $H_{i j}^{(\mathrm{VGP})}$ and $H_{k m}^{(\mathrm{VGP})}$ will be said to be directly linked, or connected, if both elements are nonzero and if the column index of the first equals the row index of the second, namely, if $j=k$. An off-diagonal matrix element $H_{l m}^{(\mathrm{VGP})}$ will be said to be reachable by $H_{i j}^{(\mathrm{VGP})}$ if there exists a sequence of directly linked off-diagonal elements $H_{i j}^{(\mathrm{VGP})} \rightarrow$ $H_{j k}^{(\mathrm{VGP})} \rightarrow \cdots \rightarrow H_{l m}^{(\mathrm{VGP})}$. Let us show that the three required conditions of reflexivity, symmetry, and transitivity are satisfied: (i) Reflexivity: Trivially $H_{i j}$ is reachable from itself. (ii) Symmetry: If $H_{i j}^{(\mathrm{VGP})}$ is reachable from $H_{l m}^{(\mathrm{VGP})}$ then the converse is also true, as the reverse sequence satisfies the

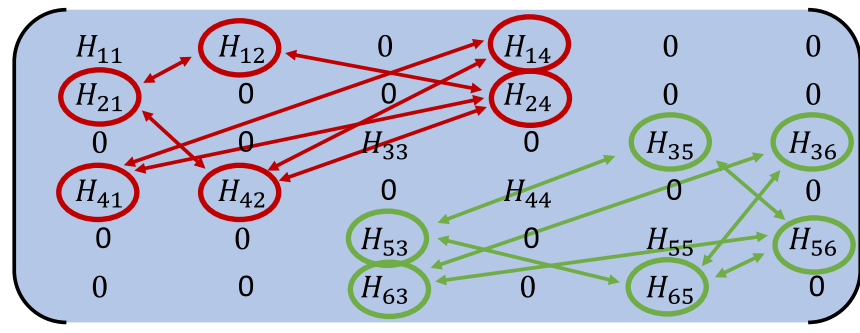

FIG. 6. Hamiltonian partitioning by reachability. An example of a $6 \times 6$ Hamiltonian containing zero elements. All the matrix elements circled in red are reachable from one another and the same holds for all elements circled in green. Elements from one group cannot be reached from those of the other. The reachability criterion partitions the Hamiltonian into two noninteracting sub-Hamiltonians.

condition as well. (iii) Transitivity: If $H_{i j}^{(\mathrm{VGP})}$ is reachable from $H_{l m}^{(\mathrm{VGP})}$ and $H_{l m}^{(\mathrm{VGP})}$ is reachable from $H_{r s}^{(\mathrm{VGP})}$, then $H_{i j}^{(\mathrm{VGP})}$ is reachable from $H_{r s}^{(\mathrm{VGP})}$ by concatenation of the two sequences. This equivalence relation allows us to split the VGP Hamiltonian matrix into noninteracting sub-Hamiltonians (see Fig. 6 for an illustration) each of which can be cured individually.

Without loss of generality, let us assume that $H^{(\mathrm{VGP})}$ has only one partition, meaning that every nonzero off-diagonal matrix element may be reached from another. We next provide a prescription for rotating $H^{(\mathrm{VGP})}$ to stoquastic form via a sequence of diagonal rotations (note that a series of sequentially applied diagonal rotations is in itself a diagonal rotation). We begin by picking a nonzero diagonal element $H_{i j}^{\text {(VGP) }}$ and applying the diagonal unitary defined by $\Theta_{1}=\left\{0, \ldots, \theta_{j}=\phi_{i j}, \ldots, 0\right\}$. The transformed Hamiltonian $e^{i \Theta_{1}} H^{(\mathrm{VGP})} e^{-i \Theta_{1}}$ is still VGP (as a unitary transformation does not affect the VGP property) and the phases of the $(i, j)$ th and $(j, i)$ th elements are now $\phi_{i j}=\phi_{j i}=0$. Denoting the transformed Hamiltonian by $H^{\text {(VGP) }}$ as well for simplicity, we next list all nonzero off-diagonal elements directly connected to $H_{i j}^{(\mathrm{VGP})}$, namely, $H_{j k}^{(\mathrm{VGP})}, H_{j l}^{(\mathrm{VGP})}, \ldots$ excluding all elements that close cycles, (i.e., lead back to indices that have already been considered, e.g., $\left.H_{j i}^{(\mathrm{VGP})}\right)$. We then apply a transformation $\Theta_{2}=\left\{0, \ldots, \theta_{k}=\phi_{j k}, \theta_{l}=\phi_{j l}, \ldots, 0\right\}$ thereby rotating to zero the phases of $H_{j k}^{(\mathrm{VGP})}, H_{j l}^{(\mathrm{VGP})}, \ldots$ (and also of $H_{k j}^{(\mathrm{VGP})}, H_{l j}^{(\mathrm{VGP})}, \ldots$ ). We proceed in a similar manner by listing all the nonzero elements connected to $H_{j k}^{(\mathrm{VGP})}, H_{j l}^{(\mathrm{VGP})}, \ldots$ that do not close cycles, i.e., $H_{k a}^{(\mathrm{VGP})}, H_{k b}^{(\mathrm{VGP})}, \ldots, H_{j c}^{(\mathrm{VGP})}, H_{j d}^{(\mathrm{VGP})}, \ldots$ curing those by an appropriate rotation $\Theta_{3}$. We continue with the process in a similar manner until all nonexcluded matrix elements are cured. Importantly, we observe that all off-diagonal matrix elements that close cycles and have therefore been excluded from curing already have zero phases by virtue of the VGP condition: Since full cycles have zero overall geometric phases and elements but the closing links have zero phases individually, having been cured already, the remaining element in each cycle must have zero phase as well.

We have thus given an explicit prescription for diagonally rotating VGP Hamiltonians to stoquastic form. 
[1] S. Bravyi, D. P. DiVincenzo, R. I. Oliveira, and B. M. Terhal, The complexity of stoquastic local Hamiltonian problems, Quant. Inf. Comp. 8, 0361 (2008).

[2] D. Aharonov and T. Naveh, Quantum NP-A survey, arXiv:quant-ph/0210077.

[3] S. Gharibian, Y. Huang, Z. Landau, and S. W. Shin, Quantum Hamiltonian complexity, Found. Trends Theor. Comput. Sci. 10, 159 (2015).

[4] T. Cubitt and A. Montanaro, Complexity classification of local hamiltonian problems, SIAM J. Comput. 45, 268 (2016).

[5] D. Landau and K. Binder, A Guide to Monte Carlo Simulations in Statistical Physics (Cambridge University Press, New York, 2005).

[6] M. E. J. Barkema and G. T. Newman, Monte Carlo Methods in Statistical Physics (Oxford University Press, New York, 1999).

[7] S. Bravyi, Monte Carlo simulation of stoquastic Hamiltonians, Quant. Inf. Comp. 15, 1122 (2015).

[8] L. Gupta and I. Hen, Elucidating the interplay between nonstoquasticity and the sign problem, Adv. Quantum Technol. 3, 1900108 (2020).

[9] L. Gupta, T. Albash, and I. Hen, Permutation matrix representation quantum Monte Carlo, J. Stat. Mech. (2020) 073105.

[10] M. Troyer and U.-J. Wiese, Computational Complexity and Fundamental Limitations to Fermionic Quantum Monte Carlo Simulations, Phys. Rev. Lett. 94, 170201 (2005).

[11] P. Henelius and A. W. Sandvik, Sign problem in Monte Carlo simulations of frustrated quantum spin systems, Phys. Rev. B 62, 1102 (2000).

[12] T. Dornheim, Fermion sign problem in path integral Monte Carlo simulations: Quantum dots, ultracold atoms, and warm dense matter, Phys. Rev. E 100, 023307 (2019).

[13] V. I. Iglovikov, E. Khatami, and R. T. Scalettar, Geometry dependence of the sign problem in quantum Monte Carlo simulations, Phys. Rev. B 92, 045110 (2015).

[14] M. Marvian, D. A. Lidar, and I. Hen, On the computational complexity of curing non-stoquastic Hamiltonians, Nat. Commun. 10, 1571 (2019).

[15] J. Klassen and B. M. Terhal, Two-local qubit Hamiltonians: When are they stoquastic? Quantum 3, 139 (2019).

[16] J. Klassen, M. Marvian, S. Piddock, M. Ioannou, I. Hen, and B. Terhal, Hardness and ease of curing the sign problem for two-local qubit Hamiltonians, arXiv:1906.08800.

[17] M. Ioannou, S. Piddock, M. Marvian, J. Klassen, and B. M. Terhal, Sign-curing local Hamiltonians: Termwise versus global stoquasticity and the use of Clifford transformations, arXiv:2007.11964.
[18] D. Hangleiter, I. Roth, D. Nagaj, and J. Eisert, Easing the Monte Carlo sign problem, Sci. Adv. 6 eabb8341 (2020).

[19] T. Albash, G. Wagenbreth, and I. Hen, Off-diagonal expansion quantum Monte Carlo, Phys. Rev. E 96, 063309 (2017).

[20] I. Hen, Off-diagonal series expansion for quantum partition functions, J. Stat. Mech. (2018) 053102.

[21] D. Joyner, Adventures in Group Theory. Rubik's Cube, Merlin's Machine, and Other Mathematical Toys (Johns Hopkins University Press, Baltimore, MD, 2008).

[22] E. T. Whittaker and G. Robinson, Divided differences, The Calculus of Observations: A Treatise on Numerical Mathematics (Dover, New York, 1967).

[23] C. de Boor, Divided differences, Surveys Approx. Theory 1, 46 (2005).

[24] N. Robertson and P. D. Seymour, Graphic Structure Theory: Proceedings of the Ams-Ims-Siam Joint Summer Research Conference on Graph Minors (American Mathematical Society, Providence, Rhode Island, 1993).

[25] D. B. West, Introduction to Graph Theory (Prentice Hall, Upper Saddle River, NJ, 2001).

[26] S. Wessel, B. Normand, F. Mila, and A. Honecker, Efficient Quantum Monte Carlo simulations of highly frustrated magnets: The frustrated spin-1/2 ladder, SciPost Phys. 3, 005 (2017).

[27] V. Ambegaokar and M. Troyer, Estimating errors reliably in Monte Carlo simulations of the Ehrenfest model, Am. J. Phys. 78, 150 (2010).

[28] R. Y. Rubinstein, Simulation and the Monte Carlo Method, 1st ed. (John Wiley \& Sons, Inc., New York, 1981).

[29] I. Hen, Resolution of the sign problem for a frustrated triplet of spins, Phys. Rev. E 99, 033306 (2019).

[30] E. Crosson, T. Albash, I. Hen, and A. P. Young, De-signing Hamiltonians for quantum adiabatic optimization, Quantum 4, 334 (2020).

[31] A. Berman and R. Plemmons, Nonnegative Matrices in the Mathematical Sciences (Society for Industrial and Applied Mathematics, Philadelphia, PA, 1994).

[32] M. Jarret, Hamiltonian surgery: Cheeger-type gap inequalities for nonpositive (stoquastic), real, and Hermitian matrices, arXiv: 1804.06857.

[33] R. Farwig and D. Zwick, Some divided difference inequalities for $n$-convex functions, J. Math. Anal. Appl. 108, 430 (1985).

[34] L. Gupta, L. Barash, and I. Hen, Calculating the divided differences of the exponential function by addition and removal of inputs, Comput. Phys. Commun. 254, 107385 (2020). 